\title{
A Portable Evanescent Wave Optic Fiber Immunosensor for Sensitive and Rapid Detection of 2,4-D in Water Samples
}

\author{
Wei Li, Jun Wu, Rong Yang, Dan Song, Feng Long \\ Renmin University of China, Beijing, China \\ Email: 1842038165@qq.com
}

How to cite this paper: $\mathrm{Li}, \mathrm{W}$., $\mathrm{Wu}, \mathrm{J}$., Yang, R., Song, D. and Long, F. (2017) A Portable Evanescent Wave Optic Fiber Immunosensor for Sensitive and Rapid Detection of 2,4-D in Water Samples. Journal of Geoscience and Environment Protection, 5, 42-45.

https://doi.org/10.4236/gep.2017.56006

Received: May 9, 2017

Accepted: June 9, 2017

Published: June 12, 2017

\begin{abstract}
A portable evanescent wave optic fiber immunosensor was developed for detection of 2,4-dichlorophenoxyacetic acid(2,4-D) using a indirect competitive immunoassay. In this paper, hapten conjugates 2,4-D-OVA were immobilized with covalent binding methods. After pre-reacting, 2,4-D-antibody-Cy5.5 in sample specifically recognized the $2,4-\mathrm{D}$ antigens binding sites on surface of the optical fiber probe. Under optimum conditions, 2,4-D could be detected in less than $18 \mathrm{~min}$ for each assay cycle. The regeneration of the optic fiber surface allowed more than 200 times without losing performance. The limits of detection of $0.039 \mathrm{ug} / \mathrm{L}$ and the quantitative detection range of $0.47-81.02$ ug/L were obtained when the concentration of 2,4-D was $1 \mathrm{mg} / \mathrm{L}$. This immunosensor shows great potential in rapid simultaneous detection of 2,4-D in waters samples.
\end{abstract}

\section{Keywords}

Evanescent Wave Optic Fiber Immunosensor, 2,4-D, Detection, Immunoassay

\section{Introduction}

2,4-Dichlorophenoxyacetic acid(2,4-D) is one of the most widely used herbicides in agriculture all over the world. It has been associated with the occurrence of cancer in humans, endocrine-disrupting activities, acute congestion and degenerative changes in central nerve system. The maximum admissible concentration for pesticides in drinking water is restricted by the European Drinking Water Act to $0.1 \mathrm{ng} / \mathrm{ml}$ for an individual pesticide and to $0.5 \mathrm{ng} / \mathrm{ml}$ for the combined total of pesticides. To satisfy the lowest level of the standard and guarantee the quality of drinking water and human health, many detection methods including HPLC, GC/MS and biosensors have been developed. The traditional de- 
tection methods require expensive instruments, skilled operators, extensive sample pretreatments and relatively large amounts of reagents ${ }^{1}$.

Compared with conventional detection methods, Evanescent wave fiber optic immunosensors (EWFI) developed to determine trace amount of 2,4-D based on the principle of immunoreaction and total internal reflect fluorescent (TIRF) has many advantages of feasible miniaturization, sensitivity, simplicity of opration, cost-effectiveness and capability of real-time rapid measurements. The stability and reusability of the sensing surface were evaluated in order to show the enormous potential of this immunosensor for more than 200 times analysis of 2,4-D.

\section{Characterization of 2,4-D-OVA Conjugate Modified Biosensor}

2,4-D-OVA conjugate, regarded as a bio-recognition molecule, was immobilized onto the surface of optic fiber probes. As shown in Figure 1, we conducted several experiments to evaluate the modification effect of biosensor. The mixture of $1 \mathrm{mg} / \mathrm{mL}$ Cy5.5 labeled anti-2,4-D antibody and PBS, $1 \mathrm{mg} / \mathrm{mL}$ Cy5.5 labeled anti-2,4-D antibody and $1000 \mathrm{ug} / \mathrm{mL}$ 2,4-D antigen and $1 \mathrm{mg} / \mathrm{mL}$ Cy5.5 labeled anti-atrazine antibody and PBS pre-reacted for $5 \mathrm{~min}$, were flowed into the optical fiber probe, respectively. When the mixture of anti-2,4-D antibody and PBS were added, the visible fluorescence signal can be examined, which suggested that the specific binding between anti-2,4-D antibody and the 2,4-D-OVA conjugate modified onto the optic fiber probes surface. When the mixture of antiatrazine antibody and PBS were added, the fluorescence signal value was tiny. It meaned the atrazine antibodies could not bind with 2,4-D-OVA conjugate immobilized onto the probe surface. When the mixture of anti-2,4-D antibody and

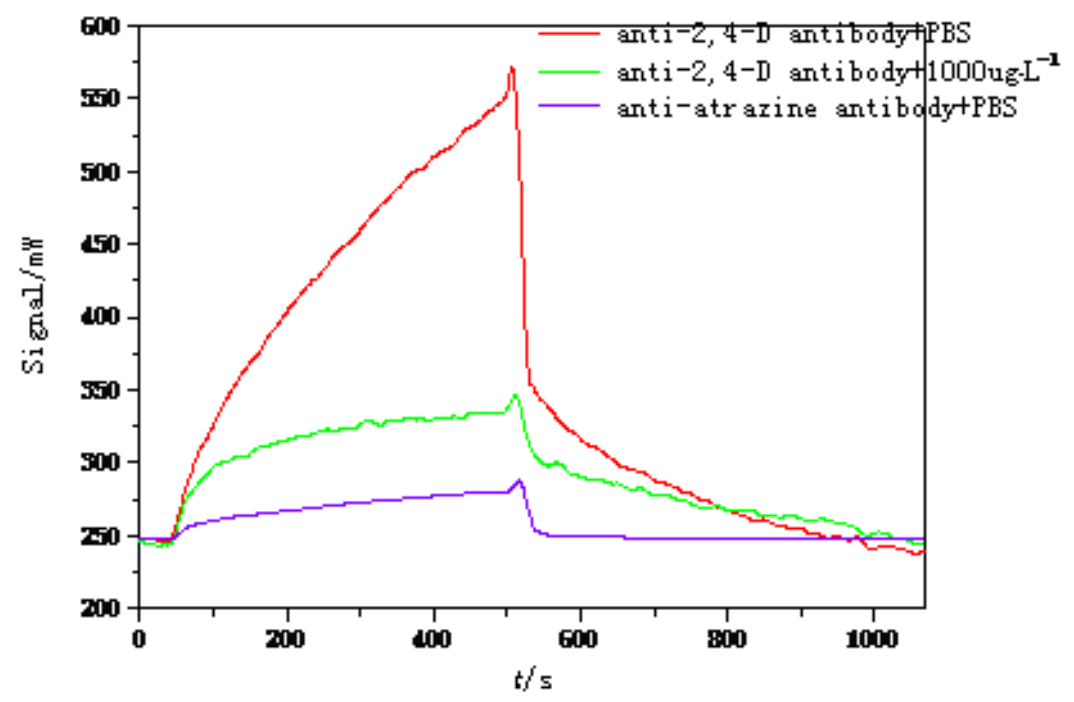

Figure 1. Assessment of the immobilization effectiveness. The mixture of Cy5.5-labeled anti-2,4-D antibody and PBS, Cy5.5-labeled anti-atrazine antibody and PBS and Cy5.5-labeled anti-2,4-D antibody and $1000 \mathrm{ug} / \mathrm{mL}$ 2,4-D antigen were pumped over the fiber optic sensor surface modified by $2,4-\mathrm{D}$-OVA, respectively. 
$1000 \mathrm{ug} / \mathrm{mL}$ 2,4-D antigen were added, the fluorescence signal can be obviously observed, but it is far less than the fluorescence signal which the mixture is 2,4-D antibody and PBS and far higher than that of adsorption of non-specific antibodies. These results demonstrated that the 2,4-D-OVA conjugate was successfully modified onto the sensor surface and had specific binding with anti-2,4-D antibody and non-specifically adsorbed with anti-atrazine antibody.

Calibration curve (Figure 2) was obtained with anti-2,4-D antibody concentration of $1 \mathrm{ug} \cdot \mathrm{mL}^{-1}$ pre-reacted with 2,4-D standard solution of various concentration in the range of $0-1000 \mathrm{ug} \cdot \mathrm{L}^{-1}$ for $4 \mathrm{~min}$. Standard curves were standardized by describing the signal of each standard point as the ratio of the maximum response. The vertical error bars represent to the standard deviation of the data points in three experiments, the standard deviations of all these data points were within $3 \%$. The $\mathrm{IC}_{50}$ value was calculated to be $5.48 \mathrm{ug} / \mathrm{L}$, the linear working range of 2,4-D was $0.47-81.02 \mathrm{ug} / \mathrm{L}$ and the limit of detection (LOD) for 2,4-D was $0.039 \mu \mathrm{g} / \mathrm{L}$. The biosensor performance meets the requirement for the determination of 2,4-D in the drinking water set by the European Union Drinking Water Directive and national standard. The entire test time is less than $20 \mathrm{~min}$ for each assay cycle.

\section{Regeneration and Biosensor Reusability}

The stability and reusability of immunosensor is critical to the successful reuse of the optical fiber probe and the accuracy of detection results, so this property was also evaluated. After the 450 s reaction between Cy5.5-labeled 2,4-D antibody and 2,4-D-OVA conjugate on the sensor surface, the biosensor was regenerated by $0.5 \%$ SDS solution ( $\mathrm{pH} 1.9$ ). As shown in Figure 3, after 200 times

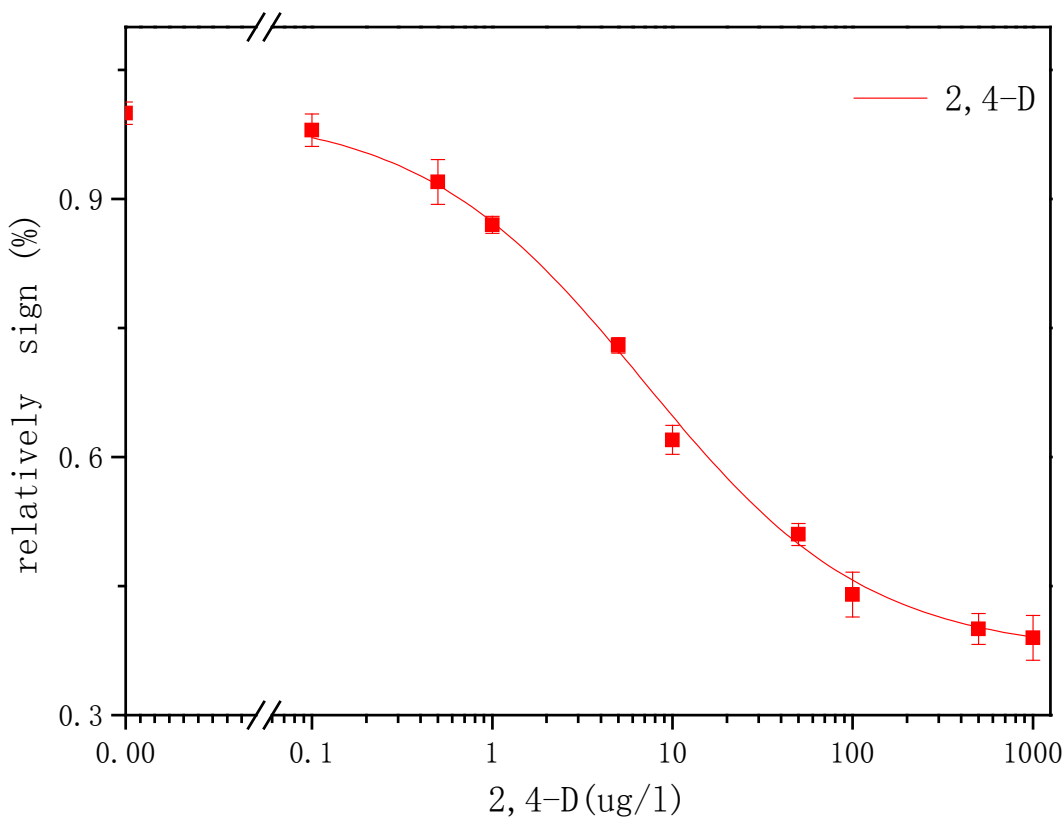

Figure 2. Standard curves of EWFI for determination of Cy5.5-labeled 2,4-D antibody concentrations of $1 \mathrm{ug} / \mathrm{mL}$. 


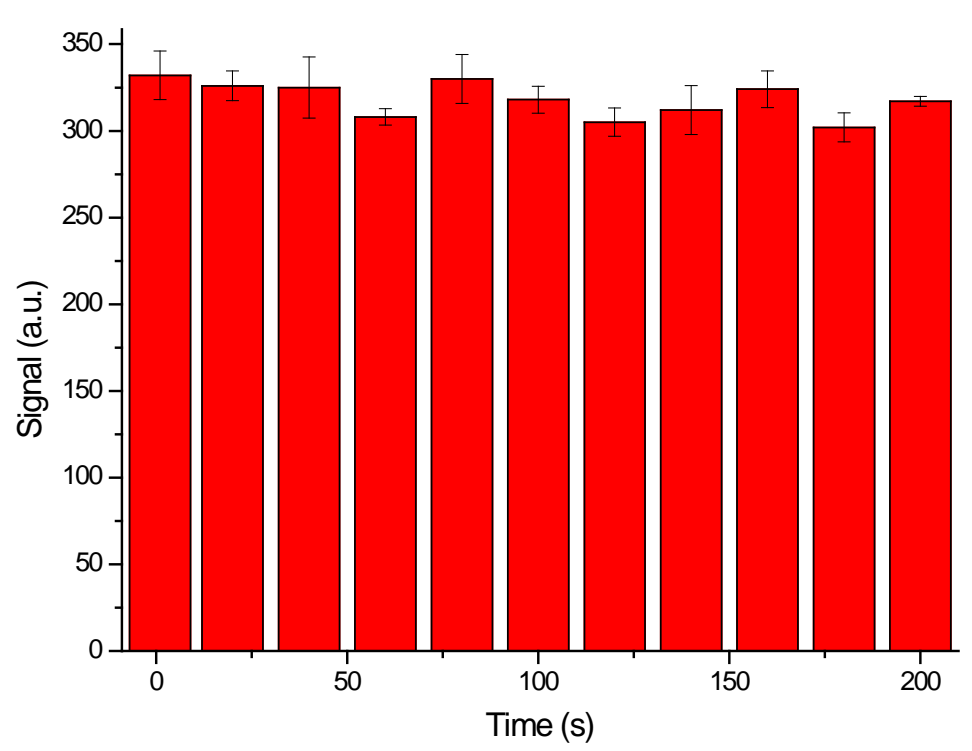

Figure 3. Signal recovery after 200 times of measurements regenerated with $0.5 \%$ SDS solution at $\mathrm{pH}=1.9$.

test assays, the optical fiber probe still maintained good performance. The signal values of all measured decreased less than $8 \%$ and the standard deviation was within $6 \%$. It indicated the regeneration of the sensor which can be used more than 200 times, thereby achieving a cost-effective and reliable 2,4-D determination.

\section{References}

[1] Long, F., Shi, H.C., He, M. and Zhu. A.N. (2008) Sensitive and Rapid Detection of 2,4-Dicholorophenoxyacetic Acid in Water Samples by Using Evanescent Wave All-Fiber Immunosensor. Biosensors and Bioelectronics, 23, 1361-1366.

https://doi.org/10.1016/j.bios.2007.12.004

[2] Lou, X.N., Zhu, A., Wang, H.L., Wu, J., Zhou, L.P. and Long, F. (2016) Direct and Ultrasensitive Optofluidic-Based Immunosensing Assay of Aflatoxin M1 in Dairy Products Using Organic Solvent Extraction. Analytica Chimica Acta, 940, 120-127. https://doi.org/10.1016/j.aca.2016.08.020

[3] Guo, H.L., Zhou, X.H., Zhang, Y., Song, B.D., Liu, L.H., Zhang, J.X. and Shi, H.C. (2014) Highly Sensitive and Rapid Detection of Melamine in Milk Products by Planar Waveguide Fluorescence Immunosensor (PWFI). Sensors and Actuators B, 194, 114-119. https://doi.org/10.1016/j.snb.2013.12.058 